\title{
Genomic footprinting with Taq polymerase
}

\author{
H. P. Saluz and J. P. Jost
}

The linear amplification of chemically sequenced DNA with DNA polymerase from Thermus aquaticus permits the direct detection of DNA methylation and protein-DNA interaction sites in vivo.

DNA methylation and protein-DNA interactions play a crucial role in gene regulation .2. Genomic sequencing pioneered by Church and Gilbert', and further refined in our laboratory ${ }^{+}$ enables the study of the methylation state of any gene in a strand- and sequencespecific manner.

When used to investigate areas of protein/DNA interaction, the technique is referred to as genomic footprinting. In this technique, cells in suspension are treated with dimethylsulphate (DMS) or ultraviolet light to modify specific DNA bases, which undergo a quantitative cleavage reaction $(\beta \text {-elimination })^{5-7}$. The modified sites are then identified by genomic sequencing. Proteins bound to DNA protect these areas from modification by DMS and subsequent chemical cleavage, leaving their "footprint" on the DNA. In contrast, ultraviolet light cleaves at sites of protein-DNA interaction.

Despite the potential of in vivo footprinting for revealing protein-DNA interactions and genomic sequencing for detecting modifications to the DNA's native state, the current procedure is rather time-consuming and complex. We sought therefore to simplify the technique and make it more accessible to less experienced users". A flow diagram of our new method is shown in Fig. $1 a$.

\section{Abbreviated strategy}

The first steps of both the old method and our new technique are the same: total genomic DNA is digested with a suitable restriction enzyme, and the resulting DNA fragments are chemically sequenced as described by Maxam and Gilbert", Fritzsche et al. ", or Rubin and Schmid". But in our technique, sequencing is followed by selective, linear amplification with the thermostable DNA polymerase from Thermus aquaticus, using a synthetic primer labelled to a very high specific radioactivity. The products of the linear amplification (Fig. 2) are then run on a sequencing gel, and the sequence information is directly obtained by exposing the dried gel to an X-ray film.

\section{A step forward}

The new technique permits sequence information to be gathered for any gene, regardless of its copy number and the size of the total genome, without electroblotting or hybridization steps (Fig. $1 b$ ). Moreover, as restriction enzymes are used only to reduce the viscosity of the genomic

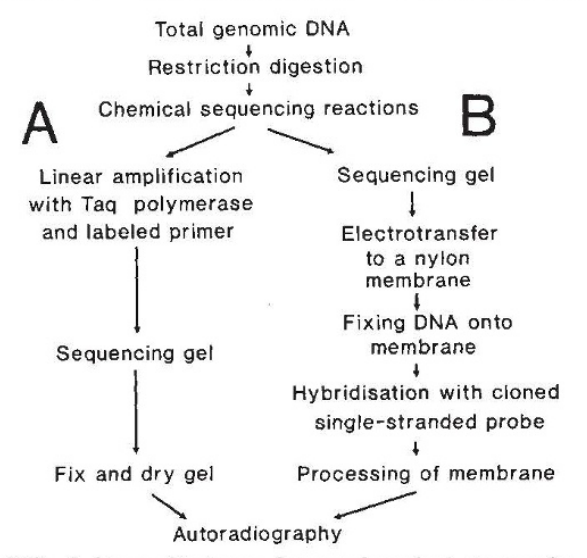

FIG. 1 Flow diagram for performing genomic experiments using the new expedited procedure $(a)$, and the classical method of genomic sequencing $(b)$.

DNA in the reaction mixture, less expensive endonucleases can be used, as long as they do not cleave within the target sequence.

But the success of our technique is highly dependent upon the purity of the Taq polymerase used, which can vary from batch to batch. The recent commercial availability of recombinant Taq polymerase should eliminate this problem. [Editor's note: Recombinantly produced Taq polymerase can be purchased from Perkin-Elmer Cetus and United States Biochemical Corporation. Purified natural Taq is sold by Perkin-Elmer Cetus, Stratagene, BRL, Beckman Instruments, and Promega.]

Our simplified procedure for studying the native state of genomic DNA relies on the linear amplification of cleaved DNA

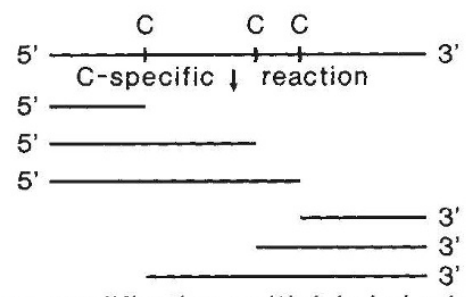

Linear amplification \ with labeled primer and Taq polymerase

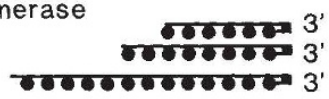

FIG. 2 A diagram illustrating the linear amplification of the sequencing reaction products. Restriction enzyme-digested DNA is first subjected to chemical sequencing reactions. The fragments generated by $\beta$-elimination are then annealed to labelled oligonucleotide primers (black squares), and the complementary strand (dotted line) is synthesized by Taq polymerase. fragments to produce many radioactively labelled copies. The resulting increase in sensitivity allows the more rapid detection of single-copy mammalian genes, and should permit the investigation of such complex genomes as those of higher plants. The technique retains all of the benefits of the original genomic sequencing procedure. The unique thermostability of Taq polymerase permits the optimal annealing of the primer to the denatured target template, and its faithful elongation. But so far, procedures for studying DNA methylation are still based on the detection of chemical cleavage products. 5-Methylcytosine reacts poorly with hydrazine, resulting in a lack of cleavage upon alkaline treatment. The position of the 5 -methylcytosine is then revealed by a gap in the sequence ladder.

Because chemical cleavage of the DNA results in fragments of different lengths, it is only possible to amplify each fragment linearly. But if procedures were available quantitatively to deaminate 5-methylcytosine residues to produce thymidine, the modified DNA could be amplified exponentially by the popularly used symmetrical amplification procedure of the polymerase chain reaction (PCR) ${ }^{12,13}$. Following dideoxy sequencing, the position of the original 5-methylcytosine would be detected by the presence of an A-T pair instead of a G-C pair in the undeaminated control DNA. We are now investigating this approach, because it would speed up the procedure enormously.

Hanspeter Saluz and Jean-Pierre Jost are at the Friedrich Miescher-Institut, PO Box 2543 , $\mathrm{CH}-4002$, Basel, Switzerland. For more information, fill in reader service number 100.

1. Razin, A. Cedar, H. \& Riggs, A.D. DNA Methylation, 392 (Springer, New York, 1984)

2. Adams, R.L.P. \& Burdon, R.H. Molecular Biology of DNA Methylation, 247 (Springer, New York, 1985).

3. Church, G.M. \& Gilbert, W. Proc. natn. Acad. Sci. U.S.A. 81. 1991-1995 (1984).

4. Saluz, H.P. \& Jost, J.P. Gene 42. 151-157 (1986); A Laboratory Guide to Genomic Sequencing. 163 (Birkhäuser, Boston, 1987)

5. Becker, P.B., Ruppert, S. \& Schütz. G. Cell $51.435-443$ (1987).

6. Saluz, H.P., Feavers, I.M., Jiricny. J. \& Jost. J.P. Proc. natn. Acad. Sci. U.S.A. 85, 6697-6700 (1988).

7. Becker, M.M. \& Wang. J.C. Nature 309. 795-798 (1984)

8. Saluz, H.P. \& Jost. J.P. Proc. natn. Acad. Sci. U.S.A. (in the press).

9. Maxam, A.M. \& Gilbert. W. Meth. Enzym. 65, 499. 560 (1980).

10. Fritzsche, E., Hayatsu, H., Igloi. G. L., lida. S. \& Kösel, H. Nucleic Acids Res. 15, 5517-5528 (1987)

11. Rubin, C.M. \& Schmid, C.W. Nucleic Acids Res. 8. 4613 $4619(1980)$

12. Saiki, R.K. et al Science 230 1350-1354 (1985).

13. Erlich. H.A., Gelfand. D.H. \& Saiki. R.K. Nature 331. 461. 462 (1988), 